\title{
Efecto de un programa de ejercicios respiratorios y aeróbicos en medio acuático versus terrestre para adultos mayores
}

\author{
The effects of a respiratory and aerobic exercises program in an \\ aquatic environment versus a land environment for elderly people
}

Nathalia Cardona Garcia ${ }^{1}$, Juan Camilo Granada Ramirez ${ }^{1}$, Mayra Alejandra Tapasco Ypia ${ }^{1}$, Stefanie Tonguino-Rosero²

Forma de citar: Cardona Garcia N, Granada Ramirez JC, Tapasco Ypia MA, Tonguino Rosero S. Efecto de un programa de ejercicios respiratorios y aeróbicos en medio acuático versus terrestre para adultos mayores. Rev Univ Ind Santander Salud. 2016; 48(4): 516-525. DOI: http://dx.doi.org/10.18273/revsal.v48n4-2016010 (c) (1)

\section{RESUMEN}

Introducción: El envejecimiento causa múltiples cambios estructurales y fisiológicos en el sistema respiratorio, lo cual predispone al desarrollo de condiciones patológicas propias de esta etapa de la vida. Eventualmente los ejercicios respiratorios son realizados en tierra, sin embargo se cree que el medio acuático ofrece mejores resultados por sus propiedades físicas favoreciendo el movimiento y la expansibilidad torácica. Objetivo: Describir el efecto de un programa de ejercicios respiratorios y aeróbicos en medio acuático versus terrestre en adultos mayores. Métodos: Estudio cuasi-experimental, longitudinal-prospectivo. Se incluyeron 28 adultos mayores de 60 años distribuidos en dos grupos: acuático y terrestre, quienes recibieron por igual un programa de ejercicios respiratorios y aeróbicos durante 10 semanas. Se realizaron mediciones antes y después de culminar el programa en la expansibilidad torácica (cirtometría), capacidad aeróbica (test de caminata 6 minutos) y calidad de vida (cuestionario WHOQOL-BREF). Resultados: Mejoría estadísticamente significativa en la expansibilidad torácica en ambos grupos, con incrementos mayores en el grupo de agua (antes 4.9 \pm 0.3 ; después $5.2 \pm 0.3 \mathrm{p}<0.001$ ). Cambios positivos de la calidad de vida en los cuatro dominios del grupo acuático: dominio psicológico (antes 58.9 \pm 11.7 ; después $69.3 \pm 8.0 \mathrm{p}=0.005$ ), salud física (antes 57.2 \pm 6.1 ; después 62.6 $\pm 7.4 \mathrm{p}=0.057$ ), relaciones sociales (antes $51.3 \pm 17.8$; después $65.7 \pm 7.8 \mathrm{p}=0.009$ ) y ambiente (antes $57.6 \pm 8.7$; después 69.7 $\pm 7.8 \mathrm{p}<0.001$ ); los datos sugieren deterioro en la dimensión salud física en el grupo terrestre (antes $65.4 \pm 12.1$; después $61.2 \pm 3.0, \mathrm{p}=0.409$ ). No hubo diferencias estadísticamente significativas en la capacidad aeróbica entre los grupos. Conclusiones: Un programa de ejercicios respiratorios y aeróbicos realizado en medio acuático y terrestre demostró un aumento significativo en la expansibilidad torácica. Sin embargo, el medio acuático podría ser el más recomendado, ya que se observó mayor incremento en la expansibilidad torácica y mejoría en la percepción de la calidad de vida.

Palabras Claves: Envejecimiento, fisioterapia, ejercicios respiratorios, ejercicio aeróbico, hidroterapia.

1. Institución Universitaria Escuela Nacional del Deporte. Cali (Colombia)

2. Institución Universitaria Escuela Nacional del Deporte. Universidad del Valle. Cali (Colombia)

Correspondencia: Mayra Alejandra Tapasco Ypia. Dirección: Carrera 41B Nº14E 06 Correo electrónico: aleja9209@hotmail.com. Teléfono: 3154493204 
ABSTRACT

Introduction: Aging causes multiple structural and physiological changes in our respiratory system, which predisposes the development of pathological conditions related to this life stage. Respiratory exercises are commonly performed on ground, nevertheless it is believed that the aquatic environment provides better results due to its physical properties that favors the movement and thoracic expansion. Objective: To describe the effect of a respiratory and aerobic program exercises in an aquatic environment versus a land environment for elderly people. Methods: Quasi-experimental study, longitudinal-prospective. 28 elderly than 60 years old were included and were distributed in two groups: aquatic and land, both groups received equitably a respiratory and aerobic exercise program during 10 weeks. Measurements were made before and after the program in the thoracic expansion (cirtometry), aerobic capacity (six minute walk test) and quality of life (WHOQOL-BREF questionnaire). Results: A statistically significant improvement in the thoracic expansion in both groups, with higher increases in the aquatic group (before 4.9 \pm 0.3 ; after $5.2 \pm 0.3 p<0.001$ ). Life quality improvement in its four domains in the aquatic group: psychological domain (before $58.9 \pm 11.7$; after 69.3 \pm 8.0 , $\mathrm{p}=0.005$ ), physical health (before 57.2 \pm 6.1 ; after 62.6 $\pm 7.4 \mathrm{p}=0.057$ ), social relationships (before 51.3 \pm 17.8 ; after $65.7 \pm 7.8, \mathrm{p}=0.009$ ) and environment (before 57.6 \pm 8.7 ; after 69.7 $\pm 7.8, \mathrm{p}<0.001$ ); the data suggest a deterioration of the physical health dimension in the land group (before $65.4 \pm 12.1$; after $61.2 \pm 3.0 \mathrm{p}=0.409$ ). There were not statistically significant differences in aerobic capacity between the groups. Conclusions: A respiratory and aerobic program exercises made in an aquatic and land environment showed a significant increase in thoracic expansion. Nevertheless, the aquatic environment might be the most recommended, as it showed a greater increase in thoracic expansion and an increased perception of quality of life by the participants.

Keywords: Aging, physical therapy specialty, breathing exercises, exercise, hydrotherapy.

\section{INTRODUCCIÓN}

Actualmente se evidencia una alta proporción de adultos mayores a nivel mundial y se estima que cada vez será mayor, se espera que para el 2050 esta cifra alcance aproximadamente los 310 millones $^{1}$. Lo anterior implica considerar el desarrollo e implementación de políticas públicas enfocadas a esta población, a fin de poder suplir sus necesidades en ámbitos socioeconómicos, sanitarios y culturales.

El proceso de envejecimiento genera múltiples cambios estructurales y fisiológicos a nivel de los diferentes sistemas corporales. Específicamente a nivel respiratorio ocasiona disminución de la retracción elástica del pulmón, disminución de la distensibilidad del tórax y disminución de la fuerza de los músculos respiratorios, lo que conlleva a una pérdida de la capacidad aeróbica y por ende a la afectación de la calidad de vida del adulto mayor ${ }^{2}$. Lo anterior demuestra factores predisponentes al desarrollo de patologías a este nivel, y por tanto se hace necesaria la búsqueda de medidas preventivas que permitan mantener y mejorar la calidad de vida relacionada con la salud de esta población. Además es de suma importancia reconocer que pese a que la prevención requiere de una gran inversión económica, ésta resulta ser más eficiente que las medidas curativas, ya que además de mejorar la calidad de vida del adulto mayor, reduce los costos sanitarios que acarrean las enfermedades respiratorias ${ }^{3}$.
El medio acuático posee unos principios hidrostáticos e hidrodinámicos que permiten la prevención y tratamiento de múltiples patologías. En el sistema respiratorio se ha demostrado que logra tonificar la musculatura torácicaabdominal, mejorar las capacidades y volúmenes pulmonares y así mismo favorece la coordinación de los movimientos respiratorios ${ }^{4}$. En los adultos mayores la realización de ejercicios en medio acuático tiene mejores resultados y mayores beneficios que los ejercicios realizados en medio terrestre en relación a condiciones osteomusculares y calidad de $\mathrm{vida}^{5,6}$, además se evidencia su relevancia para el tratamiento de patologías como asma ${ }^{7}$ y la enfermedad pulmonar obstructiva crónica $(\mathrm{EPOC})^{8}$.

Pese a lo anterior, en relación a la mecánica ventilatoria para mayores de 60 años, es escasa la literatura ${ }^{9,10}$, de esta manera la presente investigación pretende describir el efecto de un programa de ejercicios respiratorios y aeróbicos (PERA) en relación a la expansibilidad torácica, la capacidad aeróbica y la calidad de vida relacionada con la salud (CVRS) en adultos mayores de 60 años sin patologías pulmonares en medio acuático y terrestre.

\section{MATERIALES Y MÉTODOS}

El diseño del estudio fue cuasi-experimental, longitudinal prospectivo. La selección de la muestra fue no probabilística por conveniencia; se incluyeron 
dos grupos de adultos mayores físicamente activos y sin antecedentes de patologías pulmonares de una unidad recreativa en la ciudad de Cali (Colombia) entre junio y septiembre de 2015. Un grupo realizaba ejercicio en medio acuático y el otro en medio terrestre. Se vincularon 41 adultos mayores (grupo acuático $n=20$, grupo terrestre $n=21$ ), de los cuales se obtuvieron los datos sociodemográficos como: edad, género, estado civil y estrato socioeconómico.

Los criterios de inclusión usados para la selección fueron: adultos mayores de 60 años, que realizaran actividad física por más de 150 minutos por semana ${ }^{11} \mathrm{y}$ la aceptación y firma del consentimiento informado. Los criterios de exclusión fueron: antecedentes de patologías pulmonares, comorbilidades u otras complicaciones que no permitieran realizar el PERA en el agua y/o tierra como enfermedades cardiovasculares, enfermedades renales, alteraciones infecciosas o alérgicas de la piel y tres o más faltas al PERA.

Para determinar un buen estado de salud, los participantes presentaron un certificado médico y se les aplicó el PAR-Q \& YOU de la Sociedad Canadiense de Fisiología del Ejercicio $^{12}$, el cual consta de siete preguntas para detectar posibles problemas sanitarios y/o cardiovasculares que los podría excluir del estudio.

Esta investigación fue avalada por el comité de ética de la Institución Universitaria Escuela Nacional del Deporte (Cali, Colombia) (según acta 001.4, 1-03127); fue clasificado como investigación con riesgo mínimo según Articulo 11 de la resolución 008430 de 1993 del ministerio de salud. Se garantizaron todas las medidas de seguridad necesarias para la aplicación de los instrumentos de evaluación y se entregó a cada participante un consentimiento informado, donde se describió de manera detallada el propósito del estudio, riesgos y beneficios, procedimientos, duración de las intervenciones, la garantía de la confidencialidad y las circunstancias bajo las cuales se terminaría la participación en el estudio.

\section{Instrumentos de medición}

La expansibilidad torácica se evaluó mediante la cirtometría con una cinta métrica (LORD ${ }^{\circledR}$ LDC338) siguiendo el protocolo de Bockenhauer, et al. ${ }^{13}$, la cirtometría es una prueba simple y de bajo costo que ha demostrado buena confiabilidad interevaluador (variabilidad de 1.2 a $5.8 \%)^{14}$.

Para determinar el índice de masa corporal (IMC) como variable antropométrica, se midió el peso y la altura por medio de una báscula con estadiómetro (ADE M20812).
La capacidad aeróbica fue medida con el test de caminata de seis minutos (TC6M), siguiendo los parámetros establecidos por la Sociedad Americana Toracica ${ }^{15}$, esta prueba posee buena validez de constructo y fiabilidad test-retest ${ }^{16}$. Se utilizó un pasillo con una longitud de $30 \mathrm{~m}$, con dos conos en los extremos para delimitar esta distancia; antes y después de la prueba se evaluó la frecuencia respiratoria, presión arterial, saturación parcial de oxígeno y frecuencia cardiaca (pulsoximetro reliaMed model ZRPO1), además se evaluó el grado de disnea y fatiga con la escala modificada de Borg.

Finalmente, para evaluar la CVRS se solicitó el permiso de uso del cuestionario WHOQOL- BREF de la OMS ${ }^{17}$; este cuestionario está compuesto por 26 preguntas agrupadas en cuatro dominios: salud física, psicológico, relaciones sociales y ambiente. La puntuación total y de cada dominio va de 0 a 100, en donde los valores que se acercan a 100 representan una mejor CVRS y los valores que se acercan a 0 denotan una peor CVRS. Este instrumento ha demostrado tener un alto desempeño en sus propiedades psicométricas, siendo una medida fiable y valida (alfa de Cronbach $>0.8)^{18}$.

\section{Recolección de información}

Tres fisioterapeutas se entrenaron para realizar las mediciones antes y después del PERA (uno estuvo midiendo IMC y cirtrometría y los otros dos TC6M), así mismo se realizó una prueba piloto a 10 adultos mayores que compartían similares características a las requeridas para el estudio con el objetivo de probar en el campo los instrumentos de medición, después de esta prueba se adecuaron los formatos de recolección de datos y de medición para facilitar el acceso a la información.

Para la cirtometría se tuvieron en cuenta dos niveles de medición: Xifoideo (tercer espacio intercostal y décimo proceso espinoso torácico) y Axilar (apófisis xifoidea y quinto proceso espinoso torácico); se ubicó al adulto mayor en posición bípeda, con los brazos relajados para acomodar la cinta métrica sobre los referentes anatómicos y se le pidió que realizara una máxima espiración seguida de una máxima inspiración, para luego registrar en centímetros la diferencia entre los dos movimientos; se realizaron un total de tres intentos con 10 segundos de diferencia entre ellos y se eligió el mejor valor.

La medición del peso $(\mathrm{kg})$ y la talla $(\mathrm{cm})$ se realizó con los participantes en ropa ligera y sin zapatos. Para el peso la persona se ubicaba sobre la báscula mirando al frente y manteniendo los brazos relajados a cada 
lado de su cuerpo y para la talla el sujeto se ubicó de espaldas a la barra de medición y el evaluador le indicó al participante que tomará aire profundo y que lo sostuviera mientras tomaba la medición.

Durante el TC6M se incentivó al participante al inicio del test para que caminara lo más rápido, se le indicó el tiempo transcurrido durante la prueba minuto a minuto y se registró el número de metros recorridos durante la prueba. Se realizaron un total de dos pruebas para cada participante con una diferencia de 30 minutos, buscando el efecto de aprendizaje de la prueba y tomando como dato, el mayor valor recorrido en metros.
Finalmente, el cuestionario WHOQOL-BREF fue diligenciado por cada participante.

\section{Diseño y aplicación del programa de ejercicios}

Se realizó una búsqueda de la literatura en bases científicas como Medline, PEDro y SciELO, LILACS ES, con el objetivo de seleccionar artículos que incluyeran protocolos de intervención con ejercicios respiratorios y/o aeróbicos para adultos mayores. De los artículos encontrados ${ }^{8,10,19,20-25}$, se recopiló la información necesaria para diseñar el PERA utilizado en la investigación. Tabla 1.

Tabla 1. Programa de ejercicios respiratorios y aeróbicos en medio acuático y terrestre

\begin{tabular}{|c|c|c|}
\hline TIPO & DURACIÓN & DESCRIPCIÓN \\
\hline Calentamiento & 10 minutos & $\begin{array}{l}\text { - Movilidad articular para la región del cuello, tronco, } \\
\text { miembros superiores e inferiores }{ }^{8,10,20,21,22} \text {. Se realiza } 1 \text { serie de } \\
15 \text { repeticiones por cada región. } \\
\text { - Marcha estática por } 2 \text { minutos }\end{array}$ \\
\hline Ejercicios Aeróbicos & $\begin{array}{l}\text { Percepción del esfuerzo, con } \\
\text { escala de Borg modificada de } \\
\text { 3-5 puntos }{ }^{19}\end{array}$ & $\begin{array}{l}\text { - Marcha dinámica. } \\
\text { - Salto en el mismo lugar } \\
\text { - Tijeras combinadas con movimiento de miembro superior. } \\
\text { - Zancadas combinadas con movimiento de miembro superior. } \\
\text { - Barrer las manos por delante del cuerpo. } \\
\text { - Patadas- puños alternados } 8,10,21,23 \text {. } \\
\quad \text { Se realizan } 2 \text { series- } 10 \text { repeticiones. }\end{array}$ \\
\hline Ejercicios Respiratorios & 10 Minutos & $\begin{array}{l}\text { - Patrón diafragmático }{ }^{20} \\
\text { - Patrones musculares respiratorios realizados } \\
\text { progresivamente }{ }^{24,25} \\
\text { - Ventilación a nivel de la capacidad inspiratoria media y } \\
\text { máxima, realizados de forma libre y combinados con diagonales } \\
\text { de kabat D1 y D2 flexo-extensora de miembros superiores. } \\
\text { - Inspiraciones fraccionadas } \\
\text { - Suspiros Inspiratorios } \\
\text { - Espiración abreviada } \\
\text { - Inspiración a nivel del volumen residual. } \\
\quad 2 \text { series de } 5 \text { repeticiones }\end{array}$ \\
\hline Relajación & 10 Minutos & $\begin{array}{l}\text { - Caminar lentamente, realizando una respiración profunda }{ }^{25} \text {. } \\
\text { - Realizar patrón diafragmático }{ }^{20} \text {. } \\
\text { Estiramiento por } 30 \text { segundos de los principales grupos } \\
\text { musculares }{ }^{23} \text {. }\end{array}$ \\
\hline
\end{tabular}

El PERA se aplicó de la misma manera a los dos grupos y tuvo una duración de 10 semanas, con 3 sesiones semanales, para un total de 30 sesiones, cada sesión con duración de 40 minutos, distribuidos en 10 minutos de calentamiento, 10 minutos de ejercicio aeróbico, 10 minutos de ejercicios respiratorios y 10 minutos de relajación. Las intervenciones fueron dirigidas por cuatro fisioterapeutas entrenados en el PERA, dos para el grupo en medio acuático y dos para el terrestre. Para el primer grupo se utilizó una piscina rectangular de $312.5 \mathrm{~m}^{2}$ y $1.40 \mathrm{~m}$ de profundidad donde el nivel del agua estuvo ubicado aproximadamente entre la escotadura supraesternal y el tercio superior del esternón de cada participante, a una temperatura ambiente (temperatura promedio de Cali es de $25^{\circ} \mathrm{C}$ ); para el medio terrestre se contó con el kiosco principal de la unidad recreativa el cual tiene una superficie plana, antideslizante y sin presencia de obstáculos. 


\section{Análisis de datos}

Análisis descriptivo, pruebas paramétricas y noparamétricas según la distribución de cada variable fue realizado con el paquete estadístico Stata 11.0 (StataCorp. Texas, USA). La normalidad y varianza de cada variable fue determinada mediante la prueba Shapiro Wilk. Los datos descriptivos de la población de estudio, por grupo de comparación, se realizó a través de medidas de tendencia central y de dispersión; las variables cualitativas son descritas a través de frecuencias absolutas y relativas. Las comparaciones en las mediciones antes versus después por grupo fueron realizadas a través de la prueba t-student pareada, y para las comparaciones entre grupos se utilizó un análisis de covarianza ajustado por el índice de masa corporal y la presencia o no de artrosis.

\section{RESULTADOS}

La población inicial del estudio fueron 41 adultos mayores, de los cuales siete fueron excluidos (seis por comorbilidades y uno por no cumplir con la edad requisito). Al PERA ingresaron 34 adultos mayores, de los cuales 17 pertenecían al grupo acuático y 17 al grupo terrestre. Durante la intervención se excluyeron 6 adultos mayores por no tener una adecuada adherencia al programa (más de tres faltas de asistencia al PERA). Figura 1.

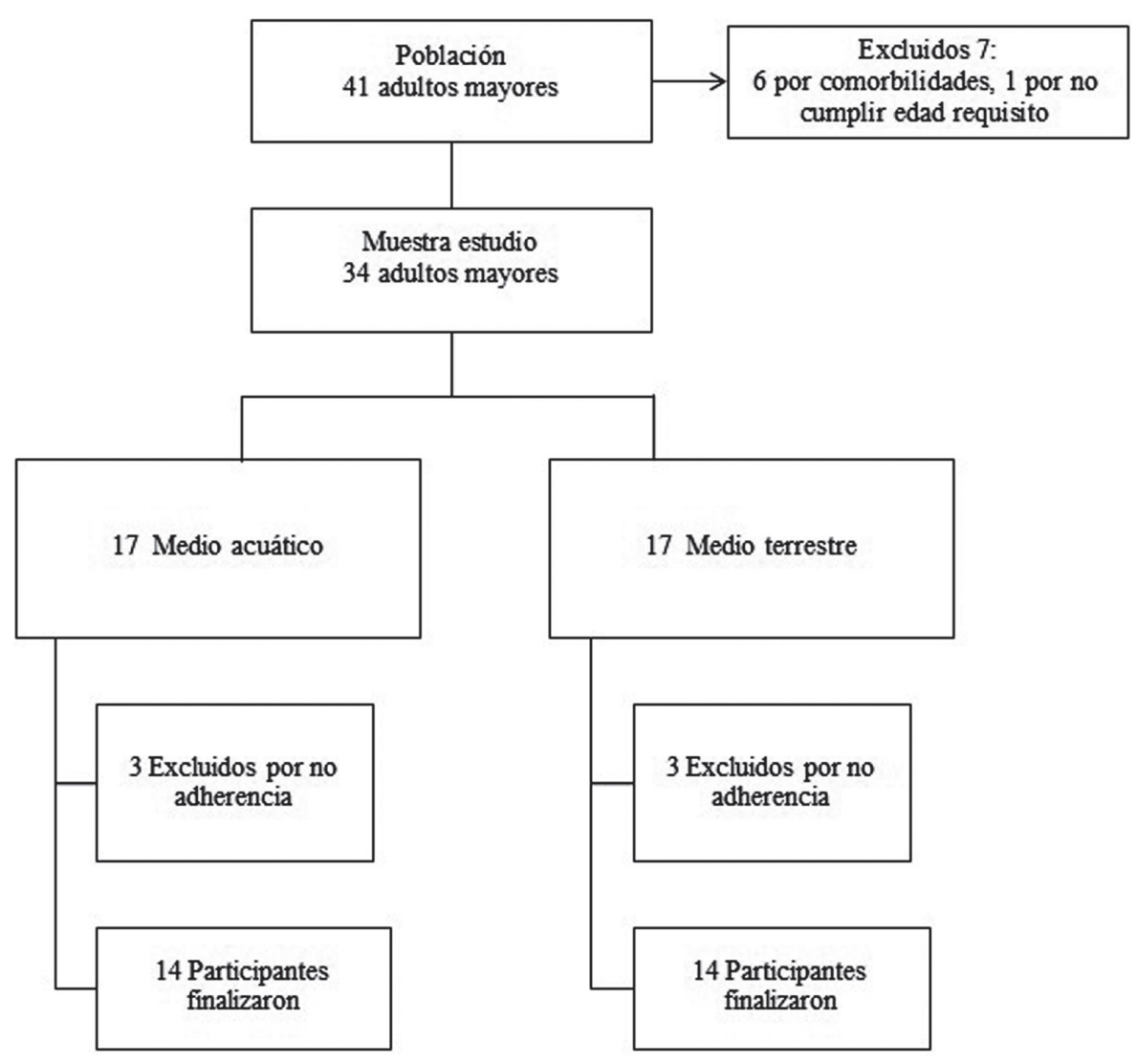

Figura 1. Descripción de la población inicial y muestra de estudio

Al evaluar las características sociodemográficasclínicas y estilos de vida no se observaron diferencias significativas en la distribución por edad, género y antecedentes personales presentes entre los grupos $(p>0.05)$. La edad promedio de los participantes fue de 74 años y se incluyó solo un hombre en el estudio, el cual perteneció al grupo acuático. La hipertensión arterial (HTA) fue el antecedente personal de mayor frecuencia, se observó una mayor proporción de artrosis $(\mathrm{p}=0.077)$ en el grupo en medio acuático y se presentó mayor peso $(\mathrm{p}=0.097)$ entre los participantes de este mismo grupo. Tabla 2.

No se encontraron diferencias en la expansibilidad torácica y en la capacidad aeróbica $(\mathrm{p}>0.05)$ al inicio del estudio. En la CVRS inicial de los participantes sólo se observaron diferencias en el dominio de salud física, siendo mayor en el grupo en medio terrestre. 
Salud Vol.48 No.4 Octubre - Diciembre de 2016

DOI: http://dx.doi.org/10.18273/revsal.v48n4-2016010

Tabla 2. Descripción de características sociodemográficas, clínicas, estilos de vida y medidas antropométricas por grupo de intervención

\begin{tabular}{|c|c|c|c|c|}
\hline Características Basales & Acuático $(n=14)$ & Terrestre $(n=14)$ & Valor P & Total $(n=28)$ \\
\hline $\begin{array}{l}\text { Edad (años) } \\
\text { Media } \pm \text { SD }\end{array}$ & $72.4 \pm 5.8$ & $75.1 \pm 6.6$ & 0.261 & $73.8 \pm 6.3$ \\
\hline $\begin{array}{l}\text { Género n (\%) } \\
\text { Masculino } \\
\text { Femenino }\end{array}$ & $\begin{array}{c}1(7.1) \\
13(92.9)\end{array}$ & $\begin{array}{c}0 \\
14(100.0)\end{array}$ & 1.000 & $\begin{array}{c}1(3.6) \\
27(96.4)\end{array}$ \\
\hline $\begin{array}{l}\text { Tiempo Actividad Física (minutos) } \\
\text { Mediana (RIQ) }\end{array}$ & $160(160-191)$ & $170(160-185)$ & 0.828 & $160(160-180)$ \\
\hline $\begin{array}{l}\text { Antecedentes n (\%) } \\
\text { Hipertensión Arterial } \\
\text { Diabetes } \\
\text { Osteoporosis } \\
\text { Artrosis }\end{array}$ & $\begin{array}{c}12(85.7) \\
3(21.4) \\
1(7.1) \\
6(42.9)\end{array}$ & $\begin{array}{c}8(57.1) \\
2(14.3) \\
3(21.4) \\
1(7.1)\end{array}$ & $\begin{array}{c}0.209 \\
1.000 \\
0.596 \\
0.077^{* *}\end{array}$ & $\begin{array}{c}20(71.4) \\
5(17.9) \\
4(14.3) \\
7(25.0)\end{array}$ \\
\hline $\begin{array}{l}\text { Peso }(\mathrm{Kg}) \\
\operatorname{Media} \pm \text { SD }\end{array}$ & $70.1 \pm 9.0$ & $63.0 \pm 12.6$ & $0.097 * *$ & $66.6 \pm 11.4$ \\
\hline $\begin{array}{l}\text { Talla }(m) \\
\text { Media } \pm \text { SD }\end{array}$ & $1.51 \pm 0.1$ & $1.54 \pm 0.1$ & 0.114 & $1.52 \pm 0.1$ \\
\hline $\begin{array}{l}\text { Índice de masa Corporal }\left(\mathrm{Kg} / \mathrm{m}^{2}\right) \\
\text { Media } \pm \text { SD }\end{array}$ & $30.9 \pm 4.4$ & $26.4 \pm 4.3$ & $0.012 *$ & $28.6 \pm 4.9$ \\
\hline
\end{tabular}

$* * \mathrm{p}<0.10,{ }^{*} \mathrm{p}<0.05 \quad$ SD: Desviación estándar RIQ: rango intercuartílico

En el grupo acuático al comparar la expansibilidad torácica antes y después de la intervención, se encontró una mejoría estadísticamente significativa, lo cual representó un aumento en la medición axilar (Antes: $4.9 \pm 0.3$, Después: $5.2 \pm 0.3, \mathrm{p}=0.000$ ) y en la medición xifoidea (Antes: 4.5 \pm 0.3 , Después: $4.8 \pm 0.3, \mathrm{p}=0.000)$. En la capacidad aeróbica, se reportó un aumento promedio respecto a la medida

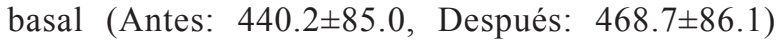
representando un incremento estadísticamente significativo $(\mathrm{p}=0.021)$. En la CVRS se encontró una mejoría después de la intervención en los cuatro dominios evaluados: salud física (diferencia 5.4 puntos), psicológica (diferencia 10.4 puntos), relaciones sociales (diferencia 14.4 puntos) y medio ambiente (diferencia 12.1 puntos).

En el grupo terrestre se observaron cambios significativos en la expansibilidad torácica axilar (Antes: 4.8 \pm 0.4 , Después: $4.9 \pm 0.4, \mathrm{p}=0.013$ ) y en la medición xifoidea (Antes: 4.5 \pm 0.4 , Después: $4.6 \pm 0.4, \mathrm{p}=0.007$ ). Se observó un cambio significativo en la capacidad aeróbica (Antes: 419.1 \pm 51.2 , Después: 454.9 \pm 27.8 ), siendo estadísticamente significativo $(\mathrm{p}=0.010)$. Finalmente no se observaron cambios en la CVRS de los participantes después de la intervención con el PERA. El dominio salud física sugiere una disminución (Inicial: 65.4 \pm 12.1, Final: $61.2 \pm 3.0, \mathrm{p}=0.409$ ). Tabla 3 .

Tabla 3. Evaluación por grupo de intervención de la expansibilidad torácica, capacidad aeróbica y CVRS antes y después del PERA

\begin{tabular}{|c|c|c|c|c|c|c|}
\hline Variables & $\begin{array}{c}\text { Medio } \\
\text { Acuático } \\
(n=14) \\
\text { Pre } \\
\text { Intervención }\end{array}$ & $\begin{array}{c}\text { Medio } \\
\text { Acuático } \\
(n=14) \\
\text { Post } \\
\text { Intervención }\end{array}$ & Valor $\mathbf{P}$ & $\begin{array}{c}\begin{array}{c}\text { Medio Terrestre } \\
(n=14)\end{array} \\
\text { Pre } \\
\text { Intervención }\end{array}$ & $\begin{array}{l}\text { Medio Terrestre } \\
\quad(n=14) \\
\text { Post Intervención }\end{array}$ & Valor $\mathbf{P}$ \\
\hline Expansibilidad Torácica Axilar & $4.9 \pm 0.3$ & $5.2 \pm 0.3$ & $0.000^{*}$ & $4.8 \pm 0.4$ & $4.9 \pm 0.4$ & $0.013 *$ \\
\hline Expansibilidad Torácica Xifoidea & $4.5 \pm 0.3$ & $4.8 \pm 0.3$ & $0.000^{*}$ & $4.5 \pm 0.4$ & $4.6 \pm 0.5$ & $0.007^{*}$ \\
\hline \multirow[t]{2}{*}{ Capacidad Aeróbica } & $440.2 \pm 85.0$ & $468.7 \pm 86.1$ & $0.021 *$ & $419.1 \pm 51.7$ & $454.9 \pm 27.8$ & $0.010^{*}$ \\
\hline & \multicolumn{6}{|c|}{ Calidad de Vida Relacionada con la Salud (CVRS) } \\
\hline Salud Física & $57.2 \pm 6.1$ & $62.6 \pm 7.4$ & $0.057 * *$ & $65.4 \pm 12.1$ & $61.2 \pm 3.0$ & 0.409 \\
\hline Psicológico & $58.9 \pm 11.7$ & $69.3 \pm 8.0$ & $0.005^{*}$ & $64.8 \pm 8.7$ & $67.4 \pm 10.8$ & 0.394 \\
\hline Relaciones Sociales & $51.3 \pm 17.8$ & $65.7 \pm 7.8$ & $0.009 *$ & $50.8 \pm 14.7$ & $52.7 \pm 17.5$ & 0.739 \\
\hline Ambiente & $57.6 \pm 8.7$ & $69.7 \pm 7.8$ & $0.000 *$ & $58.2 \pm 12.0$ & $63.3 \pm 11.8$ & $0.064 * *$ \\
\hline
\end{tabular}

$* * \mathrm{p}<0.10,{ }^{*} \mathrm{p}<0.05$ 


\section{Comparación del medio acuático versus terrestre}

Debido a las diferencias basales en los dos grupos de intervención para la comparación en las mediciones de expansibilidad torácica, capacidad aeróbica y calidad de vida relacionada con la salud se ajustó por IMC y la presencia de artrosis. Aunque al inicio del estudio los participantes intervenidos con el programa en medio acuático presentaban una mayor proporción de artrosis, índice de masa corporal y una menor puntuación en los dominios de salud física y psicológica en comparación con el grupo terrestre, los adultos mayores intervenidos con el PERA en medio acuático mostraron una mejoría de la CVRS después de la intervención en los cuatro dominios. Al comparar las diferencias en la expansibilidad torácica se encontró un incremento significativo entre los participantes del grupo acuático: axilar y xifoidea $0.3 \pm 0.2$.

Aunque en el TC6M se observó un mayor aumento de metros recorridos en el grupo terrestre (medio terrestre: $35.8 \mathrm{~m}$, medio acuático: $28.5 \mathrm{~m}$ ) esta diferencia no fue estadísticamente significativa $(\mathrm{p}=0.258)$ al ajustar por el IMC y la presencia de artrosis, puesto que estos eran mayores en el grupo acuático. Tabla 4.

Tabla 4. Comparación de las diferencias después-antes de las intervenciones para la expansibilidad torácica, capacidad aeróbica y CVRS

\begin{tabular}{lccc}
\hline \multicolumn{1}{c}{$\begin{array}{c}\text { Diferencia } \\
\text { (Después-Antes) }\end{array}$} & $\begin{array}{c}\text { Acuático } \\
(\mathbf{n}=14)\end{array}$ & $\begin{array}{c}\text { Terrestre } \\
(\mathbf{n}=\mathbf{1 4})\end{array}$ & $\begin{array}{c}\text { Valor } \\
\mathbf{P *}\end{array}$ \\
\hline $\begin{array}{l}\text { Expansibilidad Axilar }(\mathbf{c m}) \\
\text { Media } \pm \text { SD }\end{array}$ & $0.3 \pm 0.2$ & $0.1 \pm 0.1$ & $0.008^{*}$ \\
$\begin{array}{l}\text { Expansibilidad Xifoidea (cm) } \\
\text { Media } \pm \text { SD }\end{array}$ & $0.3 \pm 0.2$ & $0.1 \pm 0.2$ & 0.175 \\
$\begin{array}{l}\text { Capacidad Aeróbica (m) } \\
\text { Media } \pm \text { SD }\end{array}$ & $28.5 \pm 40.7$ & $35.8 \pm 44.6$ & 0.258
\end{tabular}

Calidad de Vida Relacionada con la Salud (CVRS)

\begin{tabular}{lccc}
$\begin{array}{l}\text { Salud Física } \\
\text { Media } \pm \text { SD }\end{array}$ & $5.3 \pm 9.6$ & $-4.3 \pm 12.2$ & $0.069^{* *}$ \\
$\begin{array}{l}\text { Psicológica } \\
\text { Media } \pm \text { SD }\end{array}$ & $10.4 \pm 11.7$ & $2.6 \pm 11.2$ & 0.132 \\
$\begin{array}{l}\text { Relaciones Sociales } \\
\text { Media } \pm \text { SD }\end{array}$ & $-12 ; 32$ & $-13 ; 19$ & \\
$\begin{array}{l}\text { Ambiente } \\
\text { Media } \pm \text { SD }\end{array}$ & $14.3 \pm 17.6$ & $1.8 \pm 20.4$ & $0.036^{*}$ \\
\hline
\end{tabular}

*Ajustado por IMC y artrosis, **p<0.10, *p<0.05, SD: Desviación estándar

\section{DISCUSIÓN}

Para este grupo de adultos mayores un PERA realizado en medio acuático y terrestre demostró cambios positivos en la expansibilidad torácica. A pesar de ello, el medio acuático podría ser el más recomendado, ya que se observó un mayor aumento en la expansibilidad torácica axilar-xifoidea y una mejoría en la percepción de la CVRS.

Los resultados de esta investigación coinciden con otros estudios de ejercicio físico en adultos mayores realizados en tierra y/o agua ${ }^{5,6,8,26}$, en donde se evidencia en las características sociodemográficas el predominio del género femenino. Según las proyecciones de población del DANE para el 2015 a nivel nacional el $54.5 \%$ de los adultos mayores de 65 años pertenecieron al género femenino y el $45.5 \%$ restante al masculino; en Cali el $59 \%$ de los adultos mayores son mujeres y el $41 \%$ hombres $^{27}$. Sumado a lo anterior, se considera que la prevalencia del género femenino en grupos de la tercera edad puede relacionarse al rol social y/o cultural que vivencian las mujeres frente a los hombres, ya que para ellas además de realizar actividad física, es más fácil compartir experiencias y preocupaciones en una comunidad ${ }^{28}$.

Son pocos los estudios que incluyen ejercicios respiratorios en medio acuático, es el caso de la investigación de Ritomy $\mathrm{M}$, et al. ${ }^{10}$, quienes encontraron similares resultados en la expansibilidad torácica axilar-xifoidea, con mejoras significativas en el grupo acuático comparado con el terrestre (AGUA axilar $1.00 \mathrm{~cm} \quad \mathrm{p}=0.03$, Xifoideo $1.37 \mathrm{~cm} \mathrm{p}=0.02$ significativo versus TIERRA axilar $2.08 \mathrm{~cm} \mathrm{p}=0.14$, Xifoideo $2.10 \mathrm{~cm} \mathrm{p}=0.25$ No significativo). Además de la expansibilidad torácica, la presente investigación tuvo en cuenta la medición de la capacidad aeróbica y la calidad de vida relacionada con la salud, lo cual evidenció cambios estadísticamente significativos en el grupo acuático.

El entrenamiento físico en medio acuático ha demostrado ser el más recomendado frente al medio terrestre, lo cual es justificado por el efecto que generan las propiedades físicas del agua sobre el cuerpo humano, ya que provocan un estado de ingravidez corporal, reduciendo el esfuerzo articular y facilitando la movilidad. Además el agua actúa como una resistencia de los movimientos corporales, que permite el fortalecimiento muscular y el mejoramiento de la mecánica respiratoria ${ }^{29}$; actuando en esta última de forma beneficiosa sobre las propiedades fisiológicas y estructurales que se ven 
afectadas durante el envejecimiento. Es así como Terán Torres M.C (2013) $)^{30}$ destaca en su estudio que el agua es un escenario que genera efectos positivos en las esferas físicas, psicológicas, sociales y ambientales, ya que mejora el autoconcepto y la autoestima de las personas al permitir realizar movimientos que en tierra no son posibles, de igual forma este medio favorece un clima social óptimo incrementando las relaciones interpersonales, sentimientos y pensamientos positivos.

La mejoría de la CVRS en medio acuático evidenciada en esta investigación, es similar a los resultados mostrados por Ramirez Velez y Triana Reina ${ }^{26}$, quienes realizaron un estudio descriptivo-transversal, aplicando un programa de actividad física terapéutica en medio acuático para un grupo de adultos mayores, en este estudio encontraron cambios positivos en la calidad de vida, probablemente generados por el bienestar corporal y mental que produce realizar actividades en el agua dado por las propiedades explicadas previamente.

Otros autores ${ }^{8,21}$, encontraron resultados semejantes en lo que concierne a la mejoría en la calidad de vida relacionada con la salud, tras la realización de ejercicios físicos en medio acuático en personas con EPOC.

Respecto a la CVRS del grupo terrestre, llama la atención que el puntaje en el dominio salud física sugiere un empeoramiento, lo cual podría ser explicado por las condiciones propias del medio terrestre, en el cual se generan limitaciones en el movimiento por acción de la fuerza de gravedad y el peso corporal. Sanders ${ }^{31}$ afirma que "realizar ejercicios en tierra genera en muchas personas dificultad al desplazarse, escaso rango de movimiento, miedo a caer, dolor, incomodidad y déficit en la forma física".

Además de loanterior, los adultos mayores pertenecientes al grupo terrestre previo a la intervención realizaban actividad física de intensidad leve, lo que corresponde a ejercicios de movilidad articular y estiramientos; al someter a esta población a un programa de ejercicios de calentamiento y ejercicio aeróbico de moderada-alta intensidad, más la inclusión de ejercicios respiratorios y de estiramiento, podría explicar el resultado del dominio físico de la calidad de vida de este grupo.

En el estudio de McNamara, et al. ${ }^{8}$ se evidencia que los ejercicios físicos en medio acuático son más eficaces en la mejora de la capacidad aeróbica en relación al medio terrestre, diferenciándose de nuestro estudio en tanto los resultados indican mejorías estadísticamente significativas en el grupo acuático y el grupo terrestre, siendo este último quien reportó mayor distancia recorrida en el TC6M. Lo anterior puede ser explicado al comparar las características basales de ambos grupos, en donde el grupo acuático registró mayor número de casos de adultos mayores con artrosis y obesidad.

En la revisión de la literatura realizada para este estudio pudimos observar que son escasos las publicaciones que incluyen el medio acuático dentro de programas de rehabilitación pulmonar, entre ellos el estudio de Rae $\mathrm{S}$, et al. ${ }^{32}$, el cual incluyó ejercicios de calentamiento, fortalecimiento y enfriamiento, además de sesiones de educación. Sin embargo, no incluyeron ejercicios respiratorios, por lo cual la presente investigación representa un aporte importante al evidenciar que después de la aplicación de un programa de ejercicios respiratorios y aeróbicos durante 10 semanas se obtienen resultados positivos en la expansibilidad torácica, capacidad aeróbica y calidad de vida relacionada con la salud. Lo anterior representa una base relevante para el desarrollo de futuras investigaciones en personas con patologías pulmonares.

Los investigadores reconocen las limitantes propias del diseño cuasiexperimental tales como el sesgo de selección (muestra tomada por conveniencia) y la falta de control sobre todas las variables (mayor proporción de artrosis e IMC alto en el grupo acuático), lo cual no permitió extrapolar los resultados a toda la población caleña de adultos mayores. A pesar de lo anterior, se tomaron medidas necesarias para contrarrestar posibles limitantes, realizando durante el análisis estadístico un ajuste por IMC y la presencia de artrosis.

Asímismoconsideramosqueeldiseñocuasiexperimental fue útil para analizar el efecto del programa de ejercicios respiratorios y aeróbicos en dos grupos de adultos mayores previamente constituidos, permitiendo tener el mayor control posible sobre las variables y facilitando el desarrollo del estudio en un ambiente natural ${ }^{33}$. Cambpell, et al. ${ }^{34}$ afirman que "precisamente por la falta de control experimental total, es imprescindible que el investigador conozca a fondo cuales son las variables particulares que su diseño especifico no controló. Así, estará más pendiente de su posible influencia y tendrá mejores elementos para evaluarla."

De acuerdo a lo anteriormente planteado se puede concluir que tras la aplicación de un PERA, el medio acuático muestra mayores cambios en el estado de la expansibilidad torácica axilar-xifoidea (promedio $0.3 \mathrm{~cm} \mathrm{p}=0.008$ ) frente al medio terrestre (promedio $0.1 \mathrm{~cm} \mathrm{p}=1.75$ ). En relación con la calidad de vida se 
reportó una mejoría en el medio acuático en los cuatro dominios de la WHOQOL-BREF, mientras que en el medio terrestre no registró cambio significativo y además la dimensión salud física evidencio en sus datos un empeoramiento. En cuanto a la capacidad aeróbica se puede concluir que no existió un cambio significativo en ambos grupos $(\mathrm{p}=0.258)$.

En consecuencia el medio acuático es el más recomendado para realizar ejercicios respiratorios y aeróbicos, sin embargo es necesaria la realización de otras investigaciones que comprueben el valor de realizar ejercicios respiratorios-acuáticos como herramienta de rehabilitación pulmonar.

\section{AGRADECIMIENTOS}

A los adultos mayores del polideportivo el Olímpico y a la Institución Universitaria Escuela Nacional del Deporte.

\section{CONSIDERACIONES ÉTICAS}

Éste estudio fue avalado por el comité de ética de la Institución Universitaria Escuela Nacional del Deporte. Los autores han obtenido el consentimiento informado de los participantes referidos en el artículo. Este documento obra en poder del autor de correspondencia por derecho a la privacidad.

\section{CONFLICTO DE INTERESES}

Los autores manifiestan no tener conflicto de interés.

\section{REFERENCIAS}

1. Organización Panamericana de la Salud (OPS). Organización Mundial de la Salud (OMS). Plan de acción sobre la salud de las personas mayores incluido el envejecimiento activo y saludable. Washington, D.C.; 2009.

2. Oyarzún M. Función respiratoria en la senectud. Rev Med Chil. 2009; 137(3): 411-8.

3. Reyes C, Silva R, Saldias F. Costo-efectividad de la rehabilitación pulmonar obstructiva crónica. Rev Chil Enf Respir. 2011; 27: 153-158.

4. Rodriguez PL, Moreno JA. Actividades acuáticas como fuente de salud. En J. A. Moreno, P. L. Rodríguez, y F. Ruiz (Eds.), Actividades acuáticas: ámbitos de aplicación. Murcia: Universidad de Murcia; 1998. P. 49-63.

5. Rua E, Silva E, Rua N. El ejercicio físico, una alternativa para mejorar la calidad de vida en el adulto mayor sedentario. Rev Cub Tec Sal. 2012; 3(3): 1-12.

6. Bergamin M, Ermolao A, Tolomio S, Berton L, Sergi $\mathrm{G}$, Zaccaria M. Water- versus land-based exercise in elderly subjects: Effects on physical performance and body composition. Clin Interv Aging. 2013; 8: 1109-1117. DOI: 10.2147/CIA.S44198.

7. Yazbek Gomiero LT, Nascimento A, Tanno LK, Agondi R, Kalil J, Giavina-Bianchi P. Respiratory exercise program for elderly individuals with asthma. Clinics (Sao Paulo). 2011; 66(7): 11631669. doi: 10.1590/S1807-59322011000700007.

8. McNamara R, McKeough Z, McKenzie D, Alison J. Water-based exercise in COPD with physical comorbidities: a randomised controlled trial. Eur Respir J. 2013; 41(6): 1284-1291. DOI: 10.1183/09031936.00034312.

9. Ide MR, Belini MA, Caromano FA. Effects of an aquatic versus non-aquatic respiratory exercise program on the respiratory muscle strength in healthy aged persons. Clinics (Sao Paulo). 2005; 60(2): 151-158. DOI: /S1807-59322005000200012.

10. Ide MR, Caromano FA, Belini MA, Guerino MR. Exercícios respiratórios na expansibilidade Torácica de Idosos: exercícios aquáticos e solo. Fisioter Movimento. 2007; 20(2): 33-40.

11. Organización Mundial de la Salud. Recomendaciones mundiales sobre actividad fisica para la salud. Suiza; 2010.

12. Canadian Society for Exercise Physiology. Physical Activity Readiness Questionnaire - PAR-Q. [Internet] 2002.

13. Bockenhauer S, Chen H, Julliard K, Weedon J. Measuring thoracic excursion: reliability of the cloth tape measure technique. J Am Osteopath Assoc. 2007; 107(5): 191-196.

14. Caldeira V, Starling C, Britto R, Martins J, Sampaio $\mathrm{R}$, Parreira V. Reliability and accuracy of cirtometry in healthy adults. J Bras Pneumol. 2007; 33(5): 519526.

15. American Thoracic Society. Guidelines for the sixminute walk test. Am J Respir Crit Care Med 2002; 166: 111-117.

16. Holland A, Spruit M, Troosters T, Puhan M, Pepin V, Saey D, et al. An official European respiratory society/American thoracic society technical standard: field walking tests in chronic respiratory disease. Eur Respir J. 2014; 44(6): 1428-1446.

17. Switzerland. Programme on Mental Health. World Health Organization. WHOQOL-BREF, Introduction, Administration, Scoring and Generic Version of the Assessment. Geneva; 1996.

18. Cardona J, Ospina L, Eljadue A. Validez 
discriminante, convergente/divergente, fiabilidad y consistencia interna, del WHOQOL-BREF y el MOSSF-36 en adultos sanos de un municipio colombiano. Rev Fac Nac Sal Pública. 2015; 33(1): 50-57.

19. ACSM (American College Sports Medicine). Guidelines for Exercise Testing and Prescription. Sixth edition. Philadelphia: Williams and Wilkins; 2000.

20. Yamaguti W, Claudino C, Neto A, Chammas M, Gomes A, Salge J, et al. Diaphragmatic breathing training program improves abdominal motion during natural breathing in patients with chronic obstructive pulmonary disease: A randomized controlled trial. Arch Phys Med Rehabil. 2012; 93(4): 571-577. DOI: 10.1016/j.apmr.2011.11.026.

21. De Souto Araujo ZT, Silva Nogueira PA, Cabral EE, dos Santos L, Silva IS, Ferreira GM. Effectiveness of low-intensity aquatic exercise on COPD: a randomized clinical trial. Respir Med 2012; 106(11): 1535-1543. DOI: 10.1016/j.rmed.2012.06.022.

22. Piazza L, Menta M, Castoldi C, Caon J, Schmidt R, Calegari L. Efeitos de exercícios aquáticos sobre a aptidão cardiorrespiratória e a pressão arterial em hipertensas. Fisioter Pesqui. 2008; 15(3): 285-291. DOI: http://dx.doi.org/10.1590/S180929502008000300012.

23. Ruoti R, Troup J, Berger R. The effects of nonswimming water exercises on older adults. J Orthop Sports Phys Ther. 1994; 19(3): 140-145. DOI: 10.2519/jospt.1994.19.3.140

24. Vieira D, Mendes L, Elmiro N, Belloso M, Britto R, Parreira V. Breathing exercises : influence on breathing patterns and thoracoabdominal motion in healthy subjects. Brazilian J Phys Ther. 2014; 18(6): 544-552. DOI: 10.1590/bjpt-rbf.2014.0048.

25. Valencia C. Calidad de vida en adultos con enfermedad pulmonar obstructiva crónica ingresados a programa de rehabilitación pulmonar. Rev Col Med Fis Rehab. 2012; 22(2): 99-108.

26. Ramirez R, Triana H. Efecto en la calidad de vida relacionada a la salud (CVRS ) de un programa de actividad física terapéutica en medio acuático, en un grupo de adultos mayores de 55 años no institucionalizado. Cali, 2006. Rev Iberoam Fisioter Kinesol. 2007; 10: 24-37. DOI: 10.1016/S11386045(07)73662-6.

27. Colombia. Demografia y población. Departamento Administrativo Nacional de Estadística (DANE). Proyecciones de población. Bogota, D.C.; 2005.

28. Mora M, Villalobos D, Araya G, Ozols A. Perspectiva subjetiva de la calidad de vida del adulto mayor, diferencias ligadas al género y a la práctica de la actividad físico recreativa. Rev $\mathrm{MH}$ Salud 2004; 1(1): 1-12.

29. Valeria J, Pourally. Kinesioterapia acuática y mecánica respiratoria. Buenos Aires, 2014. Trabajo de Grado (licenciado en Kinesiologia). Universidad FASTA. Facultad ciencias médicas.

30. Teran M. El agua, el lugar perfecto para un envejecimiento activo. Art Mov. 2010; 23-31.

31. Sanders, ME. H20 Solutions for Active Aging. IDEA Fitness J. 2010; 7(2).

32. Rae S, White P. Swimming pool-based exercise as pulmonary rehabilitation for COPD patients in primary care : feasibility and acceptability. Primary Care Respir J. 2009; 18(2): 90-94. DOI: 10.3132/ pcrj.2008.00052.

33. Londoño J.L. Metodología de la investigación epidemiológica. Editorial Universidad de Antioquia. Medellín, Colombia, 1995.

34. Campbell DT, Stanley JC. Experimental and quasiexperimental designs for research. Chicago: Rand McNally \& Company; 1996. 